\title{
Fixed Point Results on Closed Ball for a New Rational Type Contractive Mappings in Complete Dislocated Metric Spaces
}

\author{
Tahair Rasham ${ }^{1, *}$, Muhammad Arshad ${ }^{1}$, Sami Ullah Khan ${ }^{2}$ \\ ${ }^{1}$ Department of Mathematics, International Islamic University, H-10, Islamabad - 44000, Pakistan \\ ${ }^{2}$ Department of Mathematics, Gomal University D. I. Khan-29050, KPK, Pakistan \\ *Corresponding author: tahir_resham@yahoo.com \\ Received January 19, 2017; Revised March 17, 2017; Accepted April 01, 2017
}

\begin{abstract}
In this article, we find fixed point results for a pairs of mappings satisfying the locally contractive conditions on a closed ball for a new generalized rational type contraction in complete dislocated metric space. Example has been constructed to demonstrate the novelty of our results.
\end{abstract}

Keywords: fixed point, generalized rational type contraction, closed ball, complete dislocated metric spaces

Cite This Article: Tahair Rasham, Muhammad Arshad, and Sami Ullah Khan, "Fixed Point Results on Closed Ball for a New Rational Type Contractive Mappings in Complete Dislocated Metric Spaces." Turkish Journal of Analysis and Number Theory, vol. 5, no. 3 (2017): 86-92. doi: 10.12691/tjant-5-3-2.

\section{Introduction and Preliminaries}

Fixed point theory plays a fundamental role in functional analysis. Banach [4] proved significant result in the manipulation of contractive mappings in analysis. Many authors have presented a large number of theorems related to fixed point theorems. These authors have made different generalizations of the Banach's result. After that, huge number of fixed point theorems have been established by various authors and they made different generalizations of the Banach's result. Let $H: W \rightarrow W$ be a mapping. A point $w \in W$ is called a fixed point of $H$ if $w=H w$. In literature, there are many results about the fixed point of mappings that are contractive over all the theories. It is possible that $H: W \rightarrow W$ is not a contraction but $H: Y \rightarrow W$ is a contraction, where $Y$ is a closed ball in $W$. It is possible for one to get fixed point results for mappings under different condition. It has been shown by. Hussain et al. [8] related to the results concerning the presence of fixed points of a mapping that ful.lls the conditions on closed ball (see also $[1,2,3,5,15,16,17])$. The idea of dislocated is proved to be useful for logic programming semantics (see [7]). Dislocated metric space (metric-like space) (see $[10,13]$ ) is an induction of partial metric space (see [11]).

In 1994, Matthews [11] proposed the idea of partial metric spaces and got numerous fixed point results. In particular, he brought about the short connection between partial metric spaces and quasi-metric spaces, and showed a partial metric generalization of Banach's contraction mapping theorem. We can observe many findings about fixed point results on cone metric spaces (see $[9,12,14]$ ). The idea of dislocated topologies presented by Hitzler and Seda [7] is called a generalized metric a dislocated metric.
They have presented also fixed point results in complete dislocated metric spaces to draw conclusion from the well known Banach contraction principle. The aim of this paper is to strengthen the conclusion of fixed point results for a pair of mappings proving the contractive conditions on subspace for a new generalized rational type contraction in complete dislocated metric space.

Definition 1.1. [7] Let $X$ be a nonempty set. A mapping $d_{l}: X \times X \rightarrow[0, \infty)$ is called a dislocated metric (or simply $d_{l}$-metric) if the following conditions hold, for any $x, y, z \in X$ :

(1) If $d_{l}(x, y)=0$, then $x=y$;

(2) $d_{l}(x, y)=d_{l}(y, x)$;

(3) $d_{l}(x, y) \leq d_{l}(x, z)+d_{l}(z, y)$.

Then $d_{l}$ is called a dislocated metric on $X$, and the pair $\left(X, d_{l}\right)$ is called dislocated metric space or $d_{l}$ metric space. It is clear that if $d_{l}(x, y)=0$, then from (i), $x=y$. But if $x=y, d_{l}(x, y)$ may not be 0 .

Example 1.2. If $X=\mathbb{R}^{+} \cup\{0\}$, then $d_{l}(x, y)=x+y$ defines a dislocated metric on $X$.

Definition 1.3. [7] A sequence $\left\{u_{n}\right\}$ in $d_{l}$-metric space is called Cauchy sequence if for given $\varepsilon>0$, there corresponds $n_{0} \in N$ such that for all $n, m \geq n_{0}$, we have $d_{l}\left(u_{m}, u_{n}\right)<\varepsilon$.

Definition 1.4. [7] A sequence $\left\{u_{n}\right\}$ in $d_{l}$-metric space converges with respect to $d_{l}$ if there exists $u \in X$ such that $d_{l}\left(u_{n}, u\right) \rightarrow 0$ as $n \rightarrow \infty$. In this case, $u$ is called limit of $\left\{u_{n}\right\}$ and we write $u_{n} \rightarrow u$. 
Every metric space is a dislocated metric, but the converse may not be true.

Example 1.5. Let $X=R$ and $d_{l}: X \times X \rightarrow[0, \infty)$ defined by $d_{l}(u, v)=|u|+|v|$ for all $u, v \in X$.

Note that $\mathrm{dl}$ is a dislocated metric, but not a metric since $d_{l}(1,1)=2>0$.

Definition 1.6. Let $\left(X, d_{l}\right)$ be a dislocated metric space then for $u_{0} \in X, r>0$, the closed ball with centre $u_{0}$ and radius $r$ is,

$$
\overline{B_{d_{l}}\left(u_{0}, r\right)}=\left\{y \in X: d_{l}\left(u_{0}, y\right) \leq r\right\} .
$$

Definition 1.7. [7] A $d_{l}$-metric space $\left(X, d_{l}\right)$ is called complete if every Cauchy sequence in $X$ converges to a point in $X$.

Example 1.8. Let $X=[0,1]$ and $d_{l}(u, v)=\max \{u, v\}$. Then the pair $\left(X, d_{l}\right)$ is dislocated metric space, but it is not a metric space.

Definition 1.9. [7] Let $\left(X, d_{l}\right)$ be a dislocated metric space. A mapping $T: X \rightarrow X$ is called contraction if there exists $0 \leq \lambda<1$ such that

$$
d(T(u), T(v)) \leq \lambda d(u, v) \text {, for all } u, v \in X \text { with } u \neq v .
$$

Then $T$ has a unique fixed point in $X$.

The purpose of this paper is to prove common fixed point theorems for generalized rational contractions on dislocated metric spaces. We provide an example to validate our results.

\section{The Results}

In this section, we will prove the existance of common fixed points of two self mappings involving rational expressions in complete dislocated metric space.

Theorem 2.1. Let $\left(X, d_{l}\right)$ be a complete dislocated metric space and $u_{0}$ be any arbitrary point in $X$ let the mappings $S, T: X \rightarrow X$ satisfy:

$$
\begin{aligned}
d_{l}(S u, T v) \leq & a_{1} d_{l}(u, v)+a_{2} \frac{d_{l}(u, S u) \cdot d_{l}(v, T v)}{d_{l}(u, v)} \\
& +a_{3} \frac{d_{l}(u, T v) \cdot d_{l}(v, S u)}{d_{l}(u, v)} \\
& +a_{4} \frac{d_{l}(u, S u) \cdot d_{l}(v, T v)}{d_{l}(u, S u)+d_{l}(u, v)+d_{l}(v, T v)}
\end{aligned}
$$

for all $u, v \in \overline{B_{d_{l}}\left(u_{0}, r\right)}$ and $u \neq v$ with $r>0$, and

$$
d_{l}\left(u_{0}, S u_{0}\right) \leq(1-\lambda) r
$$

where $\lambda=\left(\frac{a_{1}+2 a_{3}+a_{4}}{1-a_{2}-2 a_{3}}\right)$ and $a_{1}, a_{2}, a_{3}, a_{4}$ are non negative reals with $a_{1}+a_{2}+4 a_{3}+a_{4}<1$. Then $\left\{u_{n}\right\}$ is a non increasing sequence in $\overline{B_{d_{l}}\left(u_{0}, r\right)}$ for all $n \in \mathbb{N} \bigcup\{0\}$ and $u_{n} \rightarrow h \in \overline{B_{d_{l}}\left(u_{0}, r\right)}$. Then $S$ and $T$ have common fixed point $h$ in $\overline{B_{d_{l}}\left(u_{0}, r\right)}$.

Proof: Let $u_{0}$ be an arbitrary point in $X$ and define $u_{1}=S u_{0}$ and $u_{2}=T u_{1}$ such that $d_{l}\left(u_{1}, u_{2}\right)=d_{l}\left(S u_{0}, T u_{1}\right)$.

Then

$$
\begin{aligned}
d_{l}\left(u_{1}, u_{2}\right) \leq & a_{1} d_{l}\left(u_{0}, u_{1}\right)+a_{2} \frac{d_{l}\left(u_{0}, S u_{0}\right) \cdot d_{l}\left(u_{1}, T u_{1}\right)}{d_{l}\left(u_{0}, u_{1}\right)} \\
& +a_{3} \frac{d_{l}\left(u_{0}, T u_{1}\right) \cdot d_{l}\left(u_{1}, S u_{0}\right)}{d_{l}\left(u_{0}, u_{1}\right)} \\
& +a_{4} \frac{d_{l}\left(u_{0}, S u\right) \cdot d_{l}\left(u_{1}, T u_{1}\right)}{d_{l}\left(u_{0}, S u_{0}\right)+d_{l}\left(u_{0}, u_{1}\right)+d_{l}\left(u_{1}, T u_{1}\right)} \\
& \leq a_{1} d_{l}\left(u_{0}, u_{1}\right)+a_{2} \frac{d_{l}\left(u_{0}, u_{1}\right) \cdot d_{l}\left(u_{1}, u_{2}\right)}{d_{l}\left(u_{0}, u_{1}\right)} \\
& +a_{3} \frac{d_{l}\left(u_{0}, u_{2}\right) \cdot d_{l}\left(u_{1}, u_{1}\right)}{d_{l}\left(u_{0}, u_{1}\right)} \\
& +a_{4} \frac{d_{l}\left(u_{0}, u_{1}\right) \cdot d_{l}\left(u_{1}, u_{2}\right)}{d_{l}\left(u_{0}, u_{1}\right)+d_{l}\left(u_{0}, u_{1}\right)+d_{l}\left(u_{1}, u_{2}\right)} \\
& \leq a_{1} d_{l}\left(u_{0}, u_{1}\right)+a_{2} \frac{d_{l}\left(u_{0}, u_{1}\right) \cdot d_{l}\left(u_{1}, u_{2}\right)}{d_{l}\left(u_{0}, u_{1}\right)} \\
& +a_{4} \frac{d_{l}\left(u_{0}, u_{1}\right) \cdot d_{l}\left(u_{1}, u_{2}\right)}{d_{l}\left(u_{0}, u_{1}\right)+d_{l}\left(u_{0}, u_{2}\right)} \cdot \\
& +a_{3} \frac{d_{l}\left(u_{0}, u_{2}\right) \cdot\left\{d_{l}\left(u_{1}, u_{0}\right)+d_{l}\left(u_{0}, u_{1}\right)\right\}}{d_{l}\left(u_{0}, u_{1}\right)} \\
& +a_{4} \frac{a_{1} d_{l}\left(u_{0}, u_{1}\right)+a_{2} d_{l}\left(u_{1}, u_{2}\right)+2 a_{3} d_{l}\left(u_{0}, u_{2}\right)}{d_{l}\left(u_{0}, u_{1}\right)+d_{l}\left(u_{0}, u_{1}\right)+d_{l}\left(u_{1}, u_{2}\right)} \\
& \\
& \\
& \\
&
\end{aligned}
$$

As (owing to triangular inequality),

$$
\begin{aligned}
d_{l}\left(u_{1}, u_{2}\right) \leq & a_{1} d_{l}\left(u_{0}, u_{1}\right)+a_{2} d_{l}\left(u_{1}, u_{2}\right)+2 a_{3} d_{l}\left(u_{0}, u_{1}\right) \\
& ++2 a_{3} d_{l}\left(u_{1}, u_{2}\right)+a_{4} \frac{d_{l}\left(u_{0}, u_{1}\right) \cdot d_{l}\left(u_{1}, u_{2}\right)}{d_{l}\left(u_{0}, u_{1}\right)+d_{l}\left(u_{0}, u_{2}\right)} .
\end{aligned}
$$

Where

$$
d_{l}\left(u_{1}, u_{2}\right) \leq d_{l}\left(u_{1}, u_{0}\right)+d_{l}\left(u_{0}, u_{2}\right) .
$$

Hence

$$
\begin{aligned}
d_{l}\left(u_{1}, u_{2}\right) & \leq\left(\frac{a_{1}+2 a_{3}+a_{4}}{1-a_{2}-2 a_{3}}\right) d_{l}\left(u_{0}, u_{1}\right) \\
& \leq \lambda d_{l}\left(u_{0}, u_{1}\right) \leq \lambda(1-\lambda) r, \text { by using }(2.2) \\
d_{l}\left(u_{1}, u_{2}\right) & \leq \lambda(1-\lambda) r .
\end{aligned}
$$

Where $\lambda=\frac{a_{1}+2 a_{3}+a_{4}}{1-a_{2}-2 a_{3}}$. Now,

$$
\begin{aligned}
d_{l}\left(u_{0}, u_{2}\right) & \leq d_{l}\left(u_{0}, u_{1}\right)+d_{l}\left(u_{1}, u_{2}\right) \\
& \leq(1-\lambda) r+\lambda(1-\lambda) r \\
& \leq(1-\lambda)^{2} r \leq r
\end{aligned}
$$




$$
d_{l}\left(u_{0}, u_{2}\right) \leq r
$$

This implies that $u_{2} \in \overline{B_{d_{l}}\left(u_{0}, r\right)}$. Similarly, by repeating the same process for

$$
d_{l}\left(u_{2}, u_{3}\right)=d_{l}\left(T u_{1}, S u_{2}\right)=d_{l}\left(S u_{2}, T u_{1}\right)
$$

we get

$$
d_{l}\left(u_{2}, u_{3}\right) \leq \lambda^{2} d_{l}\left(u_{0}, u_{1}\right)
$$

Consequently, $u_{3}, u_{4}, \ldots, u_{j} \in \overline{B_{d_{l}}\left(u_{0}, r\right)}$, for some $j \in N$. If $j=2 i+1$, where $i=0,1,2, \ldots, \frac{j-1}{2}$ we get

$$
d_{l}\left(u_{2 i+1}, u_{2 i+2}\right) \leq \lambda d_{l}\left(u_{2 i}, u_{2 i+1}\right) .
$$

Similarly, if $j=2 i+2$, where $i=0,1,2, \ldots, \frac{j-2}{2}$, we have

$$
d_{l}\left(u_{2 i+2}, u_{2 i+3}\right) \leq \lambda d_{l}\left(u_{2 i+1}, u_{2 i+2}\right) .
$$

Now, (2.3) implies that

$$
d_{l}\left(u_{2 i+1}, u_{2 i+2}\right) \leq \lambda^{2 i+1} d_{l}\left(u_{0}, u_{1}\right) .
$$

Also, (2.4) implies that

$$
d_{l}\left(u_{2 i+2}, u_{2 i+3}\right) \leq \lambda^{2 i+2} d_{l}\left(u_{0}, u_{1}\right) .
$$

Now, by combining (2.5) and (2.6), we have

$$
d_{l}\left(u_{j}, u_{j+1}\right) \leq \lambda^{j} d_{l}\left(u_{0}, u_{1}\right) \text { for all } j \in N
$$

Now,

$$
\begin{aligned}
d_{l}\left(u_{0}, u_{j+1}\right) & \leq d_{l}\left(u_{0}, u_{1}\right)+d_{l}\left(u_{1}, u_{2}\right)+\ldots+d_{l}\left(u_{j}, u_{j+1}\right) \\
& \leq d_{l}\left(u_{0}, u_{1}\right)+\lambda d_{l}\left(u_{0}, u_{1}\right)+\ldots+\lambda^{j} d_{l}\left(u_{0}, u_{1}\right) \\
& \quad \text { by }(2.7) \\
& \leq\left(1+\lambda+\lambda^{2}+\ldots+\lambda^{j}\right) d_{l}\left(u_{0}, u_{1}\right) \\
& \leq \frac{1\left(1-\lambda^{j}\right)}{1-\lambda}(1-\lambda) r \text { as } j \rightarrow \infty \\
& \leq r .
\end{aligned}
$$

Thus, $u_{j+1} \in \overline{B_{d_{l}}\left(u_{0}, r\right)}$. Hence $u_{n} \in \overline{B_{d_{l}}\left(u_{0}, r\right)}$ for all $n \in N$, therefore $\left\{u_{n}\right\}$ is a sequence in $\overline{B_{d_{l}}\left(u_{0}, r\right)}$. Now, the inequality (2.7) can be written as

$$
d_{l}\left(u_{n}, u_{n+1}\right) \leq \lambda^{n} d_{l}\left(u_{0}, u_{1}\right) \text { for all } n \in N .
$$

Hence for any $m>n$,

$$
\begin{aligned}
d_{l}\left(u_{n}, u_{m}\right)< & d_{l}\left(u_{n}, u_{n+1}\right)+d_{l}\left(u_{n+1}, u_{n+2}\right) \\
& +\ldots+d_{l}\left(u_{m-1}, u_{m}\right), \\
< & \left(\lambda^{n}+\lambda^{n+1}+\ldots+\lambda^{m-1}\right) d_{l}\left(u_{0}, u_{1}\right),
\end{aligned}
$$

by using (2.8)

$<\frac{\lambda^{n}}{1-\lambda} d_{l}\left(u_{0}, u_{1}\right)$.
And

$$
\begin{gathered}
d_{l}\left(u_{n}, u_{m}\right)<\frac{\lambda^{n}}{1-\lambda} d_{l}\left(u_{0}, u_{1}\right) . \\
\rightarrow 0, \text { as } m, n \rightarrow \infty
\end{gathered}
$$

This implies that $\left\{u_{n}\right\}$ is a Cauchy sequence in $\overline{B_{d_{l}}\left(u_{0}, r\right)}$. Since $\overline{B_{d_{l}}\left(u_{0}, r\right)}$ is closed and complete, there exists a point $h \in \overline{B_{d_{l}}\left(u_{0}, r\right)}$ such that $u_{n} \rightarrow h$. It follows that $h=S h$, otherwise $d(h, S h)=z>0$ and we would then have

$$
\begin{gathered}
d_{l}(h, S h) \leq d_{l}\left(h, u_{2 n+2}\right)+d_{l}\left(u_{2 n+2}, S h\right) \\
d_{l}(h, S h) \leq d_{l}\left(h, u_{2 n+2}\right)+d_{l}\left(T u_{2 n+1}, S h\right) \\
d_{l}(h, S h) \leq d_{l}\left(h, u_{2 n+2}\right)+d_{l}\left(S h, T u_{2 n+1}\right) \\
d_{l}(h, S h) \leq d_{l}\left(h, u_{2 n+2}\right)+a_{1} d_{l}\left(h, u_{2 n+1}\right) \\
+a_{2} \frac{d_{l}(h, S h) \cdot d_{l}\left(u_{2 n+1}, T u_{2 n+1}\right)}{d_{l}\left(h, u_{2 n+1}\right)} \\
+a_{3} \frac{d_{l}\left(h, T u_{2 n+1}\right) \cdot d_{l}\left(u_{2 n+1}, S h\right)}{d_{l}\left(h, u_{2 n+1}\right)} \\
+a_{4} \frac{d_{l}(h, S h) d_{l}\left(u_{2 n+1}, T u_{2 n+1}\right)}{d_{l}(h, S h)+d_{l}\left(h, u_{2 n+1}\right)+d_{l}\left(u_{2 n+1}, T u_{2 n+1}\right)} \\
\quad \leq d_{l}\left(h, u_{2 n+2}\right)+a_{1} d_{l}\left(h, u_{2 n+1}\right) \\
+a_{2} \frac{d_{l}(h, S h) \cdot d_{l}\left(u_{2 n+1}, u_{2 n+2}\right)}{d_{l}\left(h, u_{2 n+1}\right)} \\
+a_{3} \frac{d_{l}\left(h, u_{2 n+2}\right) \cdot d_{l}\left(u_{2 n+1}, S h\right)}{d_{l}\left(h, u_{2 n+1}\right)} \\
+a_{4} \frac{d_{l}(h, S h) \cdot d_{l}\left(u_{2 n+1}, u_{2 n+2}\right)}{d_{l}(h, S h)+d_{l}\left(h, u_{2 n+1}\right)+d_{l}\left(u_{2 n+1}, u_{2 n+2}\right)} .
\end{gathered}
$$

This implies that

$$
\begin{aligned}
z \leq & d_{l}\left(h, u_{2 n+2}\right)+a_{1} d_{l}\left(h, u_{2 n+1}\right)+a_{2} \frac{z \cdot d_{l}\left(u_{2 n+1}, u_{2 n+2}\right)}{d_{l}\left(u, u_{2 n+1}\right)} \\
& +a_{3} \frac{d_{l}\left(h, u_{2 n+2}\right) \cdot d_{l}\left(u_{2 n+1}, S h\right)}{d_{l}\left(h, u_{2 n+1}\right)} \\
& +a_{4} \frac{z \cdot d_{l}\left(u_{2 n+1}, u_{2 n+2}\right)}{d_{l}(h, S h)+d_{l}\left(h, u_{2 n+1}\right)+d_{l}\left(u_{2 n+1}, u_{2 n+2}\right)},
\end{aligned}
$$

which on making $n \rightarrow \infty$, gives rise $d_{l}(h, S h)=0$ a contradiction so that $h=S h$. Similarly, one can show that $h=T h$.

Example 2.2. Let $X=\mathbb{R}^{+} \cup\{0\}$ be a dislocated metric space $d_{l}: X \times X \rightarrow X$ defined by

$$
d_{l}(u, v)=\max \{u, v\} .
$$

Let $S: X \rightarrow X$ defined by

$$
S u=\left\{\begin{array}{ll}
\frac{u}{3} \text { if } & u \in[0,1] \\
2 u \text { if } & u \in(1, \infty)
\end{array}\right\} .
$$


And $T: X \rightarrow X$ defined by

$$
T v=\left\{\begin{array}{ccc}
\frac{2 v}{3} & \text { if } & u \in[0,1] \\
3 v & \text { if } & u \in(1, \infty)
\end{array}\right\} .
$$

Take $a_{1}=\frac{1}{3}, a_{2}=\frac{1}{4}, a_{3}=\frac{1}{16}, a_{4}=\frac{1}{7}, u_{0}=\frac{1}{6}, r=5$, then $\overline{B_{d_{l}}\left(u_{0}, r\right)}=[0,1] \cap X$. We have $\lambda=\left(\frac{a_{1}+2 a_{3}+a_{4}}{1-a_{2}-2 a_{3}}\right)$ $=\frac{101}{105}$ with

$$
(1-\lambda) r=\left(1-\frac{101}{105}\right) 5=\frac{4}{21}
$$

and

$$
d_{l}\left(u_{0}, S u_{0}\right)=\max \left\{\frac{1}{6}, \frac{1}{18}\right\}=\frac{1}{6}<(1-\lambda) r .
$$

Also if $u, v \in(1, \infty) \cap X$, then

$$
\begin{aligned}
d_{l}(S u, T v)= & \max \{2 u, 3 v\} \\
\geq & \frac{1}{3} \max \{u, v\}+\frac{1}{4} \frac{\max \{u, 2 u\} \cdot \max \{v, 3 v\}}{\max \{u, v\}} \\
& +\frac{1}{16} \frac{\max \{u, 3 v\} \cdot \max \{v, 2 u\}}{\max \{u, v\}} \\
& +\frac{1}{7} \frac{\max \{u, 2 u\} \cdot \max \{v, 3 v\}}{\max \{u, 3 v\}+\max \{u, v\}+\max \{v, 2 u\}} \\
\geq & a_{1} d_{l}(u, v)+a_{2} \frac{d_{l}(u, S u) \cdot d_{l}(v, T v)}{d_{l}(u, v)} \\
& +a_{3} \frac{d_{l}(u, T v) \cdot d_{l}(v, S u)}{d_{l}(u, v)} \\
& +a_{4} \frac{d_{l}(u, S u) d_{l}(v, T v)}{d_{l}(u, T v)+d_{l}(u, v)+d_{l}(v, S u)} .
\end{aligned}
$$

Taking $u=2$ and $v=3$, then clearly $u, v \in(1, \infty) \cap X$, and $u, v>1$, we have

$$
\begin{aligned}
& d_{l}(S u, T v)=\max \{2 u, 3 v\} \\
& d_{l}(S 2, T 3)=\max \{4,9\}=9 .
\end{aligned}
$$

Now,

$$
\begin{aligned}
\max \{4,9\} \geq & \frac{1}{3} \max \{2,3\}+\frac{1}{4} \frac{\max \{2,4\} \cdot \max \{3,9\}}{\max \{2,3\}} \\
& +\frac{1}{16} \frac{\max \{2,9\} \cdot \max \{3,4\}}{\max \{2,3\}} \\
& +\frac{1}{7} \frac{\max \{2,4\} \cdot \max \{3,9\}}{\max \{2,9\}+\max \{2,3\}+\max \{3,4\}} \\
\geq & \frac{1}{3} \times 3+\frac{1}{4} \frac{4 \cdot 9}{3}+\frac{1}{16} \frac{9 \cdot 4}{3}+\frac{1}{7} \frac{4 \cdot 9}{9+3+4} \\
\geq & 1+3+\frac{3}{4}+\frac{36}{112}
\end{aligned}
$$$$
9 \geq 5.0714
$$

Hence, clearly whole space does not satisfy the contractive condition. Also if $u, v \in \overline{B_{d_{l}}\left(u_{0}, r\right)}$, then

$$
\begin{aligned}
d_{l}(S u, T v)= & \max \left\{\frac{u}{3}, \frac{2 v}{3}\right\} \\
\leq & \frac{1}{3} \max \{u, v\}+\frac{1}{4} \frac{\max \left\{u, \frac{u}{3}\right\} \cdot \max \left\{v, \frac{2 v}{3}\right\}}{\max \{u, v\}} \\
& +\frac{1}{16} \frac{\max \left\{v, \frac{2 v}{3}\right\} \cdot \max \left\{v, \frac{u}{3}\right\}}{\max \{u, v\}} \\
& +\frac{1}{7} \frac{\max \left\{u, \frac{u}{3}\right\} \cdot \max \left\{v, \frac{2 v}{3}\right\}}{\max \left\{\frac{2 v}{3}\right\}+\max \{u, v\}+\max \left\{v, \frac{u}{3}\right\}} \\
\leq & a_{1} d_{l}(u, v)+a_{2} \frac{d_{l}(u, S u) \cdot d_{l}(v, T v)}{d_{l}(u, v)} \\
& +a_{3} \frac{d_{l}(u, T v) \cdot d_{l}(v, S u)}{d_{l}(u, v)} \\
& +a_{4} \frac{d_{l}(u, S u) d_{l}(v, T v)}{d_{l}(u, T v)+d_{l}(u, v)+d_{l}(v, S u)} .
\end{aligned}
$$

Hence, all contractive conditions of theorem 2.1 are satisfied.

Corollary 2.3. Let $\left(X, d_{l}\right)$ be a complete dislocated metric space and $u_{0}$ be any arbitrary point in $X$ let the mappings $S, T: X \rightarrow X$ satisfy:

$$
\begin{aligned}
d_{l}(S u, T v) \leq & a_{1} d_{l}(u, v)+a_{2} \frac{d_{l}(u, S u) \cdot d_{l}(v, T v)}{d_{l}(u, v)} \\
& +a_{3} \frac{d_{l}(u, S u) \cdot d_{l}(v, T v)}{d_{l}(u, T v)+d_{l}(u, v)+d_{l}(v, S u)}
\end{aligned}
$$

for all $u, v \in \overline{B_{d_{l}}\left(u_{0}, r\right)}$ with $r>0$, and

$$
d_{l}\left(u_{0}, S u_{0}\right) \leq(1-\lambda) r
$$

where $\lambda=\left(\frac{a_{1}+a_{3}}{1-a_{2}}\right)$ and, $a_{1}, a_{2}, a_{3}$, are non negative reals with $a_{1}+a_{2}+a_{3}<1$. If, $\left\{u_{n}\right\}$ is a non increasing sequence in $\overline{B_{d_{l}}\left(u_{0}, r\right)}$ for all $n \in \mathbb{N} \bigcup\{0\}$ and $u_{n} \rightarrow h \in \overline{B_{d_{l}}\left(u_{0}, r\right)}$. Then $S$ and $T$ have common fixed

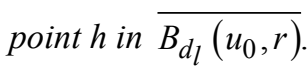

Proof: By putting $a_{3}=0$ in Theorem 2.1, we get the required result:

Theorem 2.4. Let $\left(X, d_{l}\right)$ be a complete dislocated metric space and $v_{0}$ be any arbitrary point in $X$ let the mappings $S, T: X \rightarrow X$ satisfy:

$$
d_{l}(S(v), T(f)) \leq a d_{l}(v, f)+b \frac{d_{l}(v, S(v)) d_{l}(f, T(f))}{1+d_{l}(v, f)}
$$


for all $v, f \in \overline{B_{d_{l}}\left(u_{0}, r\right)}$ and $v \neq f$ with $r>0$,

$$
d_{l}\left(v_{0}, S v_{0}\right) \leq(1-\lambda) r
$$

where $\lambda=\left(\frac{a}{1-b}\right)$ and $a, b$ are nonnegative reals with $a+b<1$. If, $\left\{v_{n}\right\}$ is a non increasing sequence in $\overline{B_{d_{l}}\left(v_{0}, r\right)}$ for all $n \in \mathbb{N} \bigcup\{0\}$ and $v_{n} \rightarrow u \in \overline{B_{d_{l}}\left(v_{0}, r\right)}$.

Then $S$ and $T$ have common fixed point $u$ in $\overline{B_{d_{l}}\left(v_{0}, r\right)}$.

Proof: Let $v_{0}$ be an arbitrary point in $X$ and define $v_{1}=S\left(v_{0}\right)$ and $v_{2}=T\left(v_{1}\right)$ such that

$$
d_{l}\left(v_{1}, v_{2}\right)=d_{l}\left(S\left(v_{0}\right), T\left(v_{1}\right)\right) \text {. }
$$

Then

$$
\begin{aligned}
d_{l}\left(v_{1}, v_{2}\right) & \leq a d_{l}\left(v_{0}, v_{1}\right)+b \frac{d_{l}\left(v_{0}, S\left(v_{0}\right)\right) d_{l}\left(v_{1}, T\left(v_{1}\right)\right)}{1+d_{l}\left(v_{0}, v_{1}\right)} \\
& \leq a d_{l}\left(v_{0}, v_{1}\right)+b \frac{d_{l}\left(v_{0}, v_{1}\right) d_{l}\left(v_{1}, v_{2}\right)}{1+d_{l}\left(v_{0}, v_{1}\right)} \\
& \leq a d_{l}\left(v_{0}, v_{1}\right)+b d_{l}\left(v_{1}, v_{2}\right)\left(\frac{d_{l}\left(v_{0}, v_{1}\right)}{1+d_{l}\left(v_{0}, v_{1}\right)}\right) \\
& \leq a d_{l}\left(v_{0}, v_{1}\right)+b d_{l}\left(v_{1}, v_{2}\right)
\end{aligned}
$$

This implies that

$$
\begin{aligned}
d_{l}\left(v_{1}, v_{2}\right) & \leq\left(\frac{a}{1-b}\right) d_{l}\left(v_{0}, v_{1}\right) \\
& \leq \lambda d_{l}\left(v_{0}, v_{1}\right) \\
& \leq \lambda(1-\lambda) r \text { by using }(2.10)
\end{aligned}
$$

Where $\left(\frac{a}{1-b}\right)=\lambda$. Now

$$
\begin{aligned}
d_{l}\left(v_{0}, v_{2}\right) & \leq d_{l}\left(v_{0}, v_{1}\right)+d_{l}\left(v_{1}, v_{2}\right), \\
& \leq(1-\lambda) r+\lambda(1-\lambda) r \\
& \leq\left(1-\lambda^{2}\right) r \leq r
\end{aligned}
$$

this implies that $v_{2} \in \overline{B_{d_{l}}\left(v_{0}, r\right)}$ similarly,

$$
\begin{aligned}
& d_{l}\left(v_{2}, v_{3}\right)=d_{l}\left(v_{3}, v_{2}\right)=d_{l}\left(S\left(v_{2}\right), T\left(v_{1}\right)\right) \\
& d_{l}\left(S\left(v_{2}\right), T\left(v_{1}\right)\right) \\
\leq & a d_{l}\left(v_{2}, v_{1}\right)+b \frac{d_{l}\left(v_{2}, S\left(v_{2}\right)\right) d_{l}\left(v_{1}, T\left(v_{1}\right)\right)}{1+d_{l}\left(v_{2}, v_{1}\right)}, \\
\leq & a d_{l}\left(v_{2}, v_{1}\right)+b \frac{d_{l}\left(v_{2}, v_{3}\right) d_{l}\left(v_{1}, v_{2}\right)}{1+d_{l}\left(v_{2}, v_{1}\right)}, \\
\leq & a d_{l}\left(v_{2}, v_{1}\right)+b d_{l}\left(v_{2}, v_{3}\right)\left(\frac{d_{l}\left(v_{1}, v_{2}\right)}{1+d_{l}\left(v_{2}, v_{1}\right)}\right), \\
& d_{l}\left(v_{2}, v_{3}\right) \leq a d_{l}\left(v_{1}, v_{2}\right)+b d_{l}\left(v_{2}, v_{3}\right) .
\end{aligned}
$$

This implies that

$$
\begin{aligned}
d_{l}\left(v_{2}, v_{3}\right) & \leq\left(\frac{a}{1-b}\right) d_{l}\left(v_{1}, v_{2}\right) \\
& \leq \lambda \cdot \lambda d_{l}\left(v_{0}, v_{1}\right) \\
& \leq \lambda^{2} d_{l}\left(v_{0}, v_{1}\right) \\
& \leq \lambda^{2}(1-\lambda) r \leq r
\end{aligned}
$$

Consequently, $v_{3}, v_{4}, \ldots, v_{j} \in \overline{B_{d_{l}}\left(v_{0}, r\right)}$, for some $j \in N$. If $j=2 i+1$, where $i=0,1,2, \ldots, \frac{j-1}{2}$ we get

$$
d_{l}\left(v_{2 i+1}, v_{2 i+2}\right) \leq \lambda d_{l}\left(v_{2 i}, v_{2 i+1}\right) .
$$

Similarly, if $j=2 i+2$, where $i=0,1,2, \ldots, \frac{j-2}{2}$, we have

$$
d_{l}\left(v_{2 i+2}, v_{2 i+3}\right) \leq \lambda d_{l}\left(v_{2 i+1}, v_{2 i+2}\right)
$$

Now, (2.11) implies that

$$
d_{l}\left(v_{2 i+1}, v_{2 i+2}\right) \leq \lambda^{2 i+1} d_{l}\left(v_{0}, v_{1}\right) .
$$

Also, (2.12) implies that

$$
d_{l}\left(v_{2 i+2}, v_{2 i+3}\right) \leq \lambda^{2 i+2} d_{l}\left(v_{0}, v_{1}\right) .
$$

Now, by combining (2.13) and (2.14), we have

$$
d_{l}\left(v_{j}, v_{j+1}\right) \leq \lambda^{j} d_{l}\left(v_{0}, v_{1}\right) \text { for all } j \in N \text {. }
$$

Now,

$d_{l}\left(v_{0}, v_{j+1}\right) \leq d_{l}\left(v_{0}, v_{1}\right)+d_{l}\left(v_{1}, v_{2}\right)+\ldots+d_{l}\left(v_{j}, v_{j+1}\right)$

$\leq d_{l}\left(v_{0}, v_{1}\right)+\lambda d_{l}\left(v_{0}, v_{1}\right)+\ldots+\lambda^{j}\left(v_{0}, v_{1}\right)$ by $(2.15)$

$\leq\left(1+\lambda+\lambda^{2}+\ldots+\lambda^{j}\right) d_{l}\left(v_{0}, v_{1}\right)$

$\leq \frac{1\left(1-\lambda^{j}\right)}{1-\lambda}(1-\lambda) r$ as $j \rightarrow \infty$

$\leq r$.

Thus $v_{j+1} \in \overline{B_{d_{l}}\left(v_{0}, r\right)}$. Hence $v_{n} \in \overline{B_{d_{l}}\left(v_{0}, r\right)}$ for all $n \in N$, therefore $\left\{v_{n}\right\}$ is a sequence in $\overline{B_{d_{l}}\left(v_{0}, r\right)}$. Now, the inequality (2.15) can be written as

$$
d_{l}\left(v_{n}, v_{n+1}\right) \leq \lambda^{n} d_{l}\left(v_{0}, v_{1}\right) \text { for all } n \in N .
$$

To prove that $\left\{v_{n}\right\}$ is a Cauchy sequence, we have for any $m>n$,

$$
\begin{aligned}
& d_{l}\left(v_{n}, v_{m}\right) \\
& \leq d_{l}\left(v_{n}, v_{n+1}\right)+d_{l}\left(v_{n+1}, v_{n+2}\right)+\ldots+d_{l}\left(v_{m-1}, v_{m}\right), \\
& \leq \lambda^{n} d_{l}\left(v_{0}, v_{1}\right)+\lambda^{n+1} d_{l}\left(v_{0}, v_{1}\right)+\ldots+\lambda^{m-1} d_{l}\left(v_{0}, v_{1}\right) \\
& \leq\left(\lambda^{n}+\lambda^{n+1}+\ldots+\lambda^{m-1}\right) d_{l}\left(v_{0}, v_{1}\right), \\
& \leq\left(\frac{\lambda^{n}}{1-\lambda}\right) d_{l}\left(v_{0}, v_{1}\right), \\
& \rightarrow 0 \text { as } m, n \rightarrow \infty .
\end{aligned}
$$


This implies that $\left\{v_{n}\right\}$ is a Cauchy sequence in $\overline{B_{d_{l}}\left(v_{0}, r\right)}$. Since $\overline{B_{d_{l}}\left(v_{0}, r\right)}$ is closed and complete, there exists a point $u \in \overline{B_{d_{l}}\left(v_{0}, r\right)}$ such that $v_{n} \rightarrow u$, and suppose $\theta=d_{l}(u, S u)$. Therefore we have

$$
\begin{gathered}
d_{l}(u, S u) \leq d_{l}\left(u, v_{2 n+2}\right)+d_{l}\left(v_{2 n+2}, S u\right) \\
=d_{l}\left(u, v_{2 n+2}\right)+d_{l}\left(T\left(v_{2 n+1}\right), S u\right) \\
=d_{l}\left(u, v_{2 n+2}\right)+d_{l}\left(S u, T\left(v_{2 n+1}\right)\right) \\
\leq d_{l}\left(v_{2 n+2}, u\right)+a d_{l}\left(u, v_{2 n+1}\right) \\
+b \frac{d_{l}(u, S u) d_{l}\left(v_{2 n+1}, T\left(v_{2 n+1}\right)\right)}{1+d_{l}\left(u, v_{2 n+1}\right)} \\
\leq d_{l}\left(v_{2 n+2}, u\right)+a d_{l}\left(u, v_{2 n+1}\right) \\
+b \frac{d_{l}(u, S u) d_{l}\left(v_{2 n+1}, v_{2 n+2}\right)}{1+d_{l}\left(u, v_{2 n+1}\right)} \\
\theta \leq d_{l}\left(u, v_{2 n+2}\right)+a d_{l}\left(u, v_{2 n+1}\right)+b \frac{\theta+d_{l}\left(v_{2 n+1}, v_{2 n+2}\right)}{1+d_{l}\left(u, v_{2 n+1}\right)} \\
\text { letting } n \rightarrow \infty, \text { and } v_{n} \rightarrow u \text { we get, } \\
(1-b) \theta \leq 0 \\
(1-b) \neq 0 \\
\theta=d_{l}(u, S u) \leq 0 .
\end{gathered}
$$

which implies that $u=S u$. It follows similarly that $u=T u$. Now, we show that $S$ and $T$ have a unique common fixed point. For this, assume that $h$ in $X$ is a second common fixed point of $S$ and $T$. Then

$$
\begin{aligned}
& d_{l}(u, h)=d_{l}(S u, T h) \\
& \leq a d_{l}(u, h)+b \frac{d_{l}(u, S u) d_{l}(h, T h)}{1+d_{l}(u, h)} \\
& \leq a d_{l}(u, h) .
\end{aligned}
$$

This implies that,

$$
\begin{array}{r}
(1-a) d_{l}(u, h) \leq 0 \\
1-a \neq 0 \\
d_{l}(u, h)=0 .
\end{array}
$$

This implies that $u=h$, completing the proof of the theorem.

Example 2.5. Let $X=\{0\} \cup \mathbb{Q}^{+}$be a dislocated metric space $d_{l}: X \times X \rightarrow X$ defined by

$$
d_{l}(v, f)=v+f .
$$

Let $S: X \rightarrow X$ defined by

$$
S(v)=\left\{\begin{array}{l}
\frac{v}{2} \text { if } v \in[0,1] \cap X \\
2 v \text { if } v \in(1, \infty) \cap X
\end{array}\right\} .
$$

And $T: X \rightarrow X$ defined by

$$
T(f)=\left\{\begin{array}{c}
\frac{3 f}{4} \text { if } f \in[0,1] \cap X \\
5 f \text { if } f \in(1, \infty) \cap X
\end{array}\right\} .
$$

Take $a=\frac{1}{3}, b=\frac{1}{4}, v_{0}=\frac{1}{6}, r=5, \quad$ then $\overline{B_{d_{l}}\left(v_{0}, r\right)}$

$=[0,1] \cap X$. We have $\lambda=\left(\frac{a}{1-b}\right)=\frac{4}{9}$ with

$$
(1-\lambda) r=\left(1-\frac{4}{9}\right) 5=\frac{25}{9}
$$

and

$$
d_{l}\left(v_{0}, S v_{0}\right)=\frac{1}{6}+\frac{1}{12}=\frac{1}{4}<(1-\lambda) r .
$$

Also if $v, f \in(1, \infty) \cap X$, then

$$
\begin{aligned}
d_{l}(S(v), T(f)) & =2 v+5 f \\
& \geq \frac{1}{3}(v+f)+\frac{1}{4} \frac{(v+2 v) \cdot(f+5 f)}{1+(v+f)} \\
& \geq \frac{1}{3}(v+f)+\frac{9 v f}{2\{1+(v+f)\}} \\
d_{l}(S(v), T(f)) & \geq a d_{l}(v, f)+b \frac{d_{l}(v, S(v)) d_{l}(f, T(f))}{1+d_{l}(v, f)} .
\end{aligned}
$$

Then $v=2$ and $f=3$, then clearly $v, f \in(1, \infty) \cap X$, and $v, f \geq 1$, we have

$$
\begin{aligned}
& 19 \geq \frac{5}{3}+\frac{1}{4} \times \frac{54}{12} \\
& 19 \geq 2.785 .
\end{aligned}
$$

Hence, clearly whole space does not satisfy the contractive condition. Also if $v, f \in \overline{B_{d_{l}}\left(v_{0}, r\right)}$, then

$$
\begin{aligned}
& d_{l}(S(v), T(f)) \\
& =\frac{v}{2}+\frac{3 f}{4} \leq \frac{1}{3}(v+f)+\frac{1}{4} \frac{\left(v+\frac{v}{2}\right) \cdot\left(f+\frac{3 f}{4}\right)}{1+(v+f)} \\
& d_{l}(S(v), T(f)) \\
& \leq a_{l}(v, f)+b \frac{d_{l}(v, S(v)) d_{l}(f, T(f))}{1+d_{l}(v, f)} .
\end{aligned}
$$

Hence, the contractive conditions of Theorem (2.4) are satisfied.

As an application of Theorems (2.1) and (2.4) we prove the following theorem for two finite families of mappings.

Theorem 2.6. If $\left\{T_{p}\right\}_{1}^{m}$ and $\left\{S_{p}\right\}_{1}^{n}$ are two finite pair wise commuting finite families of self mappings de.ned on complete dislocated metric space $\left(X, d_{l}\right)$ such that the mappings $T$ and $S$ satisfy the conditions of theorems (2.1) and (2.4), then the component maps of the two families $\left\{T_{p}\right\}_{1}^{m}$ and $\left\{S_{p}\right\}_{1}^{n}$ have a unique common fixed point. 
Proof. In view of theorems (2.1) and (2.4), one can infer that $T$ and $S$ have a unique common fixed point $q$ i.e. $T q=S q=q$. Now we are required to show that $q$ is common fixed point of all the components maps of both the families. In view of pairwise commutativity of families of $\left\{T_{p}\right\}_{1}^{m}$ and $\left\{S_{p}\right\}_{1}^{n}$, (for every $1 \leq i \leq m$ ) we can write

$$
T_{i} q=T_{i} S q=S T_{i} q \text { and } T_{i} q=T_{i} T q=T T_{i} q
$$

which shows that $T_{i} q$ (for every $i$ ) is also a common fixed point of $T$ and $S$. By using the uniqueness of common fixed point, we can write $T_{i} q=q$ (for every $i$ ) which shows that $q$ is the common fixed point of the family $\left\{T_{p}\right\}_{1}^{m}$. Using the foregoing arguments, one can also shows that (for every $1 \leq i \leq n$ ) $S_{i} q=q$. This completes the proof of the theorem.

Corollary 2.7. If $S: X \rightarrow X$ be a self mapping de.ned on a complete dislocated metric space $\left(X, d_{l}\right)$ satisfying the condition

$$
\begin{aligned}
d_{l}(S u, S v) \leq & a d_{l}(u, v)+b \frac{d_{l}(u, S u) \cdot d_{l}(v, S v)}{d_{l}(u, v)} \\
& +c \frac{d_{l}(u, S v) \cdot d_{l}(v, S u)}{d_{l}(u, v)} \\
& +d \frac{d_{l}(u, S u) \cdot d_{l}(v, S v)}{d_{l}(u, S v)+d_{l}(u, v)+d_{l}(v, S u)}
\end{aligned}
$$

for all $u, v \in \overline{B_{d_{l}}\left(u_{0}, r\right)}$ with $r>0$,

$$
d_{l}\left(u_{0}, S u_{0}\right) \leq(1-\lambda) r
$$

where $\lambda=\left(\frac{a+2 c+d}{1-b-2 a_{3}}\right)$ and $a, b, c, d$ are non negative reals with $a+b+4 c+d<1$. Then $\left\{u_{n}\right\}$ is a non increasing sequence in $\overline{B_{d_{l}}\left(u_{0}, r\right)}$ for all $n \in \mathbb{N} \cup\{0\}$ and $u_{n} \rightarrow h \in \overline{B_{d_{l}}\left(u_{0}, r\right)}$. Then $S$ has common fixed point $h$ in $\overline{B_{d_{l}}\left(u_{0}, r\right)}$.

Corollary 2.8. If $S: X \rightarrow X$ be a self mapping defined on a complete on dislocated metric space $\left(X, d_{l}\right)$ satisfying the condition:

$$
d_{l}(S u, S v) \leq a_{1} d_{l}(u, v)+a_{2} \frac{d_{l}(u, S u) \cdot d_{l}(v, S v)}{d_{l}(u, v)}
$$

for all $u, v \in \overline{B_{d_{l}}\left(u_{0}, r\right)}$ with $r>0$,

$$
d_{l}\left(u_{0}, S u_{0}\right) \leq(1-\lambda) r
$$

where $\lambda=\left(\frac{a_{1}}{1-a_{2}}\right) a_{1}$ and $a_{2}$ are non negative reals with $a_{1}+a_{2}<1$. If, $\left\{u_{n}\right\}$ is a non increasing sequence in $\overline{B_{d_{l}}\left(u_{0}, r\right)}$ for all $n \in \mathbb{N} \bigcup\{0\}$ and $u_{n} \rightarrow h \in \overline{B_{d_{l}}\left(u_{0}, r\right)}$ Then $S$ has common fixed point $h$ in $\overline{B_{d_{l}}\left(u_{0}, r\right)}$.

\section{Conflict of Interests}

The authors declare that they have no competing interests.

\section{References}

[1] M. Arshad, A. Shoaib, and P. Vetro, Common Fixed Points of a Pair of Hardy Rogers Type Mappings on a Closed Ball in Ordered Dislocated Metric Spaces, Journal of Function Spaces, 2013 (2013), Article ID 63818.

[2] M. Arshad, A. Shoaib, M. Abbas and A. Azam, Fixed Points of a pair of Kannan Type Mappings on a Closed Ball in Ordered Partial Metric Spaces, Miskolc Mathematical Notes, 14(3), 2013, 769-784.

[3] M. Arshad, A. Azam, M. Abbas and A. Shoaib, Fixed point results of dominated mappings on a closed ball in ordered partial metric spaces without continuity U.P.B. Sci. Bull., Series A, 76(2), 2014.

[4] S. Banach, Sur les operations dans les ensembles abstraits et leur application aux equations int egrales, Fund. Math., 3 (1922), 133-181.

[5] I. Beg, M. Arshad, A. Shoaib,Fixed Point on a Closed Ball in ordered dislocated Metric Space, Fixed Point Theory, 16(2), 2015.

[6] P. Hitzler, Generalized metrics and topology in logic programming semantics. $\mathrm{PhD}$ thesis, National University of Ireland (University College, Cork) (2001).

[7] P. Hitzler, A. K. Seda, Dislocated topologies. J. Electr. Eng. 51(12), 3-7 (2000).

[8] N. Hussain, M. Arshad, A. Shoaib and Fahimuddin, Common Fixed Point results for $\alpha-\psi$-contractions on a metric space endowed with graph, J. Inequalities and Appl., 2014, 2014: 136.

[9] S. U. Khan, A. Bano. Common .xed point theorems in Cone metric spaces using W-distance, Int. J. of Math. Anal, Vol. 7, 2013, no. 14, 657-663.

[10] E. Karapınar, H. Piri, and H. H. Alsulami, Fixed points of modi.ed F-contractive mappings in complete metric-like spaces, J. Funct. Spaces, Volume 2015, Article ID 270971, 9 pages.

[11] S.G.Matthews, Partial metric topology Proc. 8th Summer Conference on General Topology and Applications, Ann. New York Acad. Sci., vol. 728, 1994, pp. 183-197.

[12] S. Radenovic, B. E. Rhoades, Fixed point theorem for two non-self mappings in cone metric spaces. Comput. Math. Appl. 57 1701-1707 (2009).

[13] Y. Ren, J. Li, and Y. Yu, Common Fixed Point Theorems for Nonlinear Contractive Mappings in Dislocated Metric Spaces, Abstr. Appl. Anal., 2013 (2013), Article ID 483059, 5 pages.

[14] S. Rezapour, R. Hamlbarani, Some notes on the paper .Cone metric spaces and fixed point theorems of contractive mappings.. J. Math. Anal. Appl. 345 719-724 (2008).

[15] A. Shoaib, M. Arshad and J. Ahmad, Fixed point results of locally cotractive mappings in ordered quasi-partial metric spaces, The Scientific World Journal, 2013 (2013), Article ID 194897, 8 pages.

[16] A. Shoaib, M. Arshad and MA. Kutbi, Common .xed points of a pair of Hardy Rogers Type Mappings on a Closed Ball in Ordered Partial Metric Spaces, J. Comput. Anal. Appl., 17(2014), 255-264.

[17] A. Shoaib, $\alpha-\eta$ Dominated Mappings and Related Common Fixed Point Results in Closed Ball, Journal of Concrete and Applicable Mathematics, 13(1-2), 2015, 152-170. 